I'(RI.88642

PREPRINT

Con-82116.6--1

A REVIEW OF QUANTUM MONTE CARLO METHDOS AND RESULTS FOR COULOMBIC SYSTEMS

D. Ceperley

This paper was prepared for submittal to Conference Proceedings

Monte Carlo Methods in Quantum Problems

November 29-December 3, 1982

Paris, France

january 27,1983

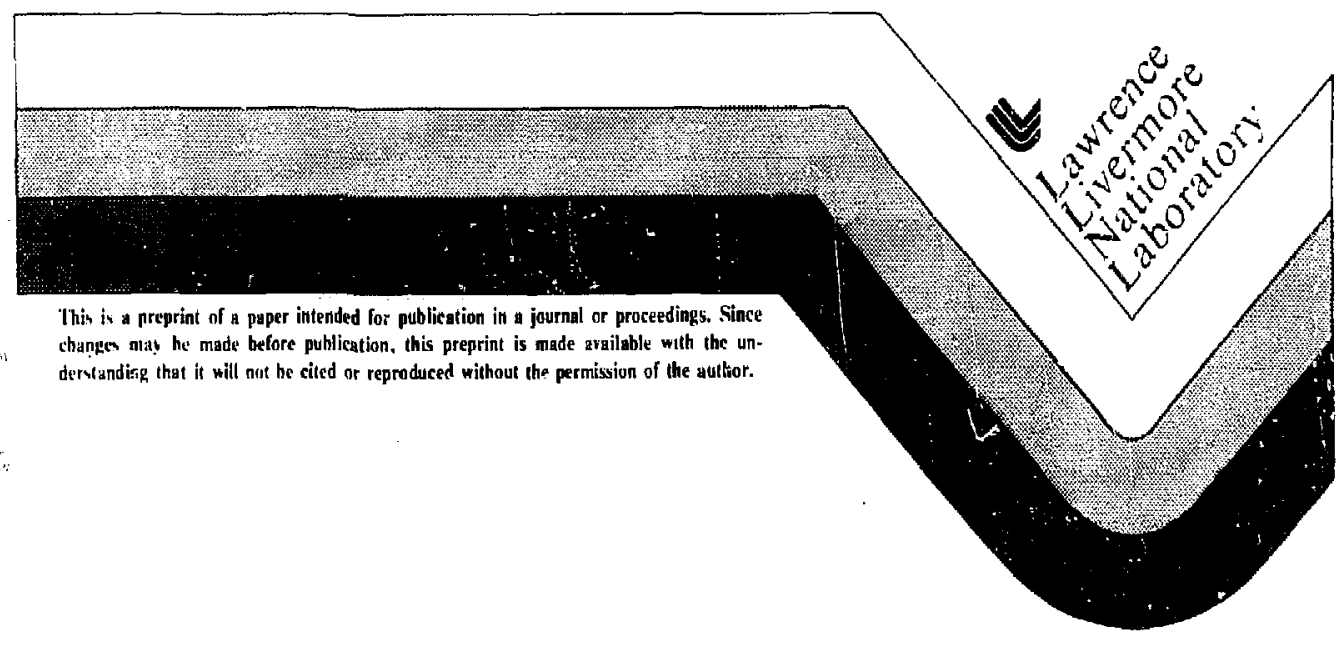

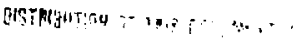


UCRL $=-88642$

DE83 006570

\title{
A REVIEN OF QUANTUM MONTE CARLO METHOOS AND RESULTS FOR COULOMBIC SYSTEMS
}

\author{
D. Ceperley \\ Lawrence Livermore National Laboratory \\ Livermore, California 94550 USA
}

The various Monte Carlo methods for calculating ground state energies are briefly reviewed. Then a summary of the charged systems that have been studied with Monte Carlo is giveri. These include the electron gas, small molecules, a metal slab and many-body hydrogen.

\section{A. INTROOUCTION}

Despite the importance of Coulombic systems, an accurate and practical computational method for determining the properties of charged quantum many-body systems is still lacking, although certainly much progress has been made. In recent years there have been several attempts to apply stochastic or Monte Carlo methods to this problem, with success for simple systems. This article will give a brief review of hese methods, the results that have been obtained so far, and give some indication of what future progress may be anticipated.

Quantum charged systems are particularly challenging for Monte Carlians, firstly because there has been so much previous research with other numerical methods that the level of accuracy needed to become competitive is rather high, and sacondly, because to answer many important questions in materials science or cnemistry, what is needed are accurate relative energies, whereas Monte Carlo errors are typically relative to the total energies. Luckily, as will be explained below, what is known about the wavefunction from other methods can be :sed to reduce the statistical errors. Thus in a rough sense, Monte Carlo can be viewsd as a way of taking a wavefunction derived in sons

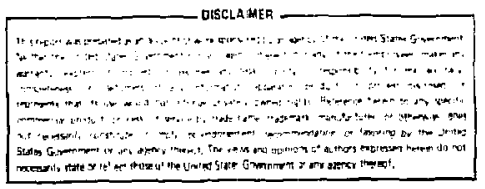


other way and reducing its errors by several orders of magnitude. First, the various Monte Carlo methods used for quantum systems will be described in order of increasing accuracy and complexity, then the results will be surveyed.

\section{B. METHODS}

1. Variational Monte Carlo

Variational Monte Carlo is simply the use of the Metropolis algorithm (I) (of course other algorithms (2) could and have been used) to compute the value of the many body integrals which arise in the variational bound to the ground state energy with a given trial function $\psi T$. The variational energy car be written in the form

$$
E_{V}=\int d^{3 n_{R}} P(R)\left[\psi_{T}^{-1} \cdot \mathscr{K}^{C} \psi_{T}\right]
$$

where $P(R)$ is the normalized distribution

$$
P(R)=\left|\psi_{T}(R)\right|^{2} / \int d^{3} n_{R}\left|\psi_{T}(R)\right|^{2}
$$

and $\mathscr{H}$ is the many-body Hamiltonian. Points $\{R\}$, can be sampled with a Markov chain using the Metropolis rejection technique. This was first applied by McMillan (3), (independently by Levesque (4l) to calculate an upper bound to the ground state energy of liquid helium four using a Jastrow trial function. For fermion systems $\psi_{T}$ must be antisymmetric. This is usually aucomplished by multiplying the Jastrow function a Slater determinant of one body orbitals. The computational algerithm for handling such determiriants efficiently was first considered by Ceperley (5).

The only peculiarity of Coulombic systems from the Monte Carlo point of view, is their long range interaction which implies that boundary conditions must be chosen carefully. It is found necessary for accurate calculations in both classical and quantum simulations, to use fully periodic boundary conditions, which means the bare Coulomb potential acting between two charges is replaced by the Ewald image potential, which is the sum of the interaction of a charge with all of the images of the other charge in the periodically replicated system. Ceperley (6) applied the variational method to the fermion one comprnent plasma (alsu known as the electron gas or jellium) and obtaineid gord agrefinert with other theoretical approaches.

The so-called "zero-variance" property of the ground state energy can be seen fron Eq. (1). As $\psi T$ approaches an exact 
eigenstate, the local energy $[\mathcal{H} \psi T / \psi T]$ approaches a constant, the energy of this state. Then the fluctuations in the estimate of $E$ will diminish dramatically. In practice this means that physical or chemical knowledge of $\psi$ can be used to reduce the error bar on the estimate of the energy. Monte Carlo can use very unconventional forms for the trial function since the standard requirement of analytic integrability no longer applies. The sole consideration is the speed with which the trial function and its derivatives can be computed. As yet this flexibility has been exploited very little.

\section{Diffusion Monte Carlo}

The Diffusion Monte Carlo (OMC) in contrast to Variational Monte Carlo, can potentially compute the exact ground state energy, at least for boson systems. The role of the trial wavefunction changes from determining an energy bound to guiding the random walk, thereby making it more efficient. One begins by mulitiplying the Schrödinger equation in imaginary time by a trial function. After some manipulations, the master equation for the stochastic process with importance sampling is:

$$
\frac{d f(R, t)}{d t}=-\left(\psi_{T}^{-1}, \mathscr{H} \psi_{T}-E_{T}\right) f+\frac{\hbar^{2}}{2 m} \nabla\left(\nabla f-2 f \psi_{T}{ }^{-1} \nabla \psi_{T}\right)
$$

where $E_{T}$ is the trial energy. It is easy to show that the stationary solution for $\boldsymbol{f}$ is $\psi T \varphi$ where $\varphi$ is the ground state eigenfunction and that the ground state energy is given by Eq. (1) with $P(R)$ proportional to $f(R, t)$. The interpretation of Eq. (3) as a stochastic process goes as follows: The second term on the RHS conserves probability as can be seen by integrating $\mathrm{Eq}_{\mathrm{q}}$. (3) with respect to $\mathrm{R}$. Let the "population" be defined as:

$$
Q(t)=\int d R f(R, t)
$$

then using Green's theorem.

$$
\frac{d Q(t)}{d t}=-\int d R\left[\psi_{T}^{-1} \mathscr{H}_{T}-E_{T}\right] f \quad .
$$

Then changes in the population came from the instantaneous values of the local energy relative to the trial energy. This is accomplished in the algorithm by branching: creating either zero, one or two replicas of a given point depending on the local energy. Thus in quantum Monte Carlo one works with an ensemble of systems. The population is stabilized when $E_{T}$ equals the mean value of the local energy, which will be equal in the large time limit to the exact ground state energy. The second term in Eq. (3) is simply diffusion with drift: i.e., 
the Smoluchowski equation in the theory of Brownian motion. To numerically simulate Eq. (3) with the OMC algorithm one makes a short time approximation (7) to the Green's function for this equation. Thus the results are only exact in the limit that the time step becomes sufficiently small.

One can see from Eq. (5) that the crucial role of importance sampling is to reduce the fluctuations in branching. If there were no importance sampling, i.e., if $\psi T=1$, then the branching would be proportional to the bare potential. Since potential energy is unbourided above and below for charged particles, the population would fluctuate greatly, and in fact, many body simulations would be impossible. towever simply putting the correct cusp conditions (7) in the trial wavefunction makes the local energy bounded, at least for bosons.

\section{The Fixed-Node Approximation}

The requirement that the wavefunction for fermions be antisymmetric is a serious difficulty for the Diffusion Mante Carlo algorithm. Unless $\psi_{T}$ has the same sign everywhere as $\varphi F$ (the fermion ground state) then $f=\psi T \varphi F$ will change signs and hence camnot be interpreted as a probability. But this amounts to knowing the nodal surfaces of $4 \mathrm{~F}$, which is very difficult in three dimensions (but not in one dimension). There are essentially two approaches to solving this problem: converting the fermion system to a boson system in some assumed subvolume of the entire space or assigning positive or negative weights to the random walks and thereby build up negative distributions in some regions. In the fixed node approximation (8) the nodes of an antisymnetric trial function $\psi T$, become an absorbing barrier to the random walk, i.e., the boundary condition $\varphi=0$ when $\psi T=0$ is imposed. This is accomplished in the algorithm in a very simple fashion, whenever $\psi T(R)$ changes sign in the course of a walk, that walk is deieted from the ensemble. It is easy to show that the energy as estimated from Eq. (1), will be an upper bound to the fermion ground state energy (9), in fact the hest upper bound with the given nodal constraint. Results to date on small molecules, show in fact that the use of the nodes of very crude trial wavefunctions yields typically $90 \%$ of the correlation energy. The fixed-node energy seems to be insensitive to the choice of the nodes.

\section{Nounl Relaxation}

There are several ways of going beyond the fixed node approximation, all of them involve random walks which carry a negative weight and suffer from serious limitations when applied to many-alectron systerns. Kalos, elsewhere in this 
volume, discusses some of these in more ditail. The only approach that has been tried for charged systems is called nodal relaxation (8), since the idea is to use the distribution generated by the fixed node algorithm and relax the nodal constraint for a short time. The exact fermion distrioution (i.e., UFFF) is then the asymptotic difference between two distributions, that resulting from random walks which cross the nodes of $\psi_{T}$ an even number of times und that from an odd number of crossings. The difficulty with this methoo is immediately apparent. After a time such that random walks have on the average crossed a node several times, the difference between these two distributions wili be largely random. The signal to noise ratio decreases exponentially, with an exponent given by the fermi minus bose energy. For this reason it is called a transient estimate of the ground state energy since one cannot in practice follow the process for large times. $A^{2}$ best, one can hope to correct the fixed-node distribution locally.

Figure 1 shows the results of applying nodal relaxation to the molerule $\mathrm{LiH}$ using three different trial functions, as a function of time since the nodes were released. Correlated sampling (9) has been used to estimate the difference between the fixed-node and release-node energy more accurately than either one separately. This difference is sensitive mainly to the energy of the walks which actually cross a node. Thus the difference in energy shown in Fig. 1 is significant. The error bars on the difference is $10^{-3}$ a.u. The three trial functions are minimal besis set floating Gaussian functions, $\psi_{1}$ was optimized while $\psi_{2}$ and $\psi_{3}$ were "de-optimized" just to raise their fixed-node energy. Although the error bars depend significantly on the trial function, all three energies relax to within one standard ercor of the experimental energy.

Similar relaxation studies have been carried out for the electron gas, where is has been found that the Hartree-fock nodes are quite accurate. In contrast, in líquid helium three, nodal relaxation has not yet achieved the experimental energy, possibly indicating that strong interactions significantly alter the nodal surfaces.

\section{Green's Function Monte Carlo}

It was realized many years ago that an integral version of the Schrodinger equation can be modeled exactly by a stochastic process. The time step errors which arise in DMC are unnecessary aporoximations. This Green's Function Monte Carlo (GFMC) algorithm is very similar to OMC. The new elements in the procedure are the introduction of a guiding approximate Greer's function, the use of a variahle time step and the 


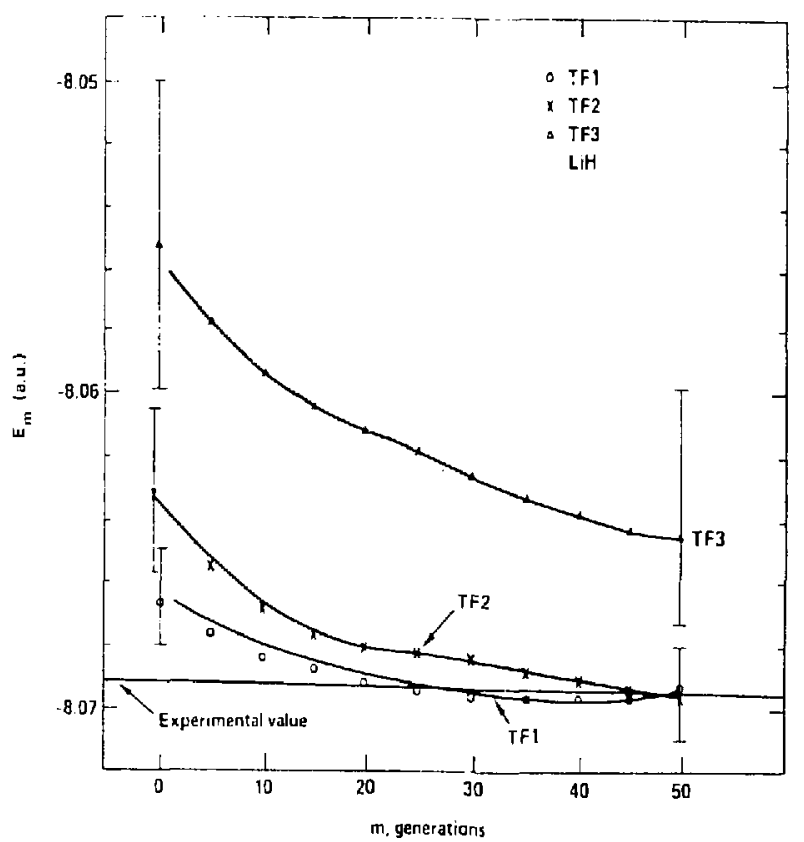

Figure 1

generation of intermediate points, points on the random walk which do not contribute to averages. This method is reviewed in Ref. 10. The first successful application of this algorithm to a many-body problem was by Kalos, Levesque, end verlet (KLV) for the case of quantum hard spheres (11). When applied directly to Coulombic system the KLV algorithrn gives very small timesteps, about $1 \%$ as far as in DMC. Recently the KLV algorithm has been generalized (12), essentially to allow for a better approximate Green's function. A good Green's function can be obtained by putting togetrer exact two-body Green's functions, just as is done with the trial function. Because of a spherial symmetry possessed by the coulomb potential, such two-body Green's functions can be conveniently tabulated. This new algorithm then allows electrons to move past each other in a single step, the algorithm is then exact, but with a time step similar to that in DMC.

\section{RESIJLTS \\ 1. Small Molecules}

The ground state energies of several small molecules have 
been calculated using all of the above Monte Carlo techniques (7). These molecules include $\mathrm{H}_{2}, \mathrm{He}_{1} \mathrm{He}_{2}, \mathrm{LiH}, \mathrm{Li}_{2}$ and $\mathrm{H}_{2} \mathrm{O}$. The fixed node approximation using very crude trial functions obtains at least $90 \%$ of the correlation energy. However, the statistical error appears to increase rapidly with the ruclear charge. For $\mathrm{H}_{2}$ or $\mathrm{LiH}$ it is approxinately $10^{-3}$ a.u. (assuming $10^{5}$ samples) while for $\mathrm{H}_{2} \mathrm{O}$ it is $10^{-2}$ a.u. Hence, simple Monte Carlo methods will have limited utility unless much better trial functions can be found. See the article in this volume by Moskowitz and Ref. (7) for a more detailed account of these calculations.

\section{The One-Component Plasma}

The one-component plasma with Fermi statistics is the familiar jellium model of solid state physics which models the conduction band of a simple metal by smearing out the ions to form a uniform positive background. At densities appropriate to metals $\left(2 \leq \mathrm{r}_{S} \leq 6\right)$ the Monte Carlo energies agree well with the results for several other sophist:cated many-body theories (8), in fact, they are often now taken as a benchmark. At lower densities $\left(r_{s}>10\right.$ ) where electron correlation becomes dominant these other theories become less accurate. The simulations have established that the plasna undergoes Wigner crystallization at a density $T_{\mathrm{S}}=100$. In addition, the fixed-node results show that a partially polarized electron gas has a lower energy in the density range $20 \leq \mathrm{I}_{\mathrm{S}} \leq 100$ than the normally unpolarized or paramagnetic electron fluid. However, the Monte Carlo variational results of Levesque (see this volune) warn that this ferromagnetism may be an artifact of the fixed-node approximation. The nodes of a polarized Slater determinant may be more accurate than those of an unpolarized Slater determinant, hence fixed-node calculations may favor the polarized phase. Further investigation of this issue is in progress, as well as consideration of more complicated types of spin ordering. In addition to the three dimensional fermion calculations, there are unpublished calculations on fermion and boson systems in both two and three dimensions.

\section{Metallic Surface}

A simple generalization of the jellium model, is to place the electrons in an inhomogenemus background. Recently Johnson (13) has conoleted a study of clortrons trapped in a potential well in the $z$ dirstion but free in the other two dimensions. This is a simple model of a thin metal film with the ions being replaced by a step function background. Figure 2 shows a comparison of the electron density as obtained by variational and fixed-node Monte Carlo and by density functional theory at 
the background density $r_{S}=5$. There are differences on the order of $10 \%$ in the surface region with the fixed-node profile being significantly sharper.

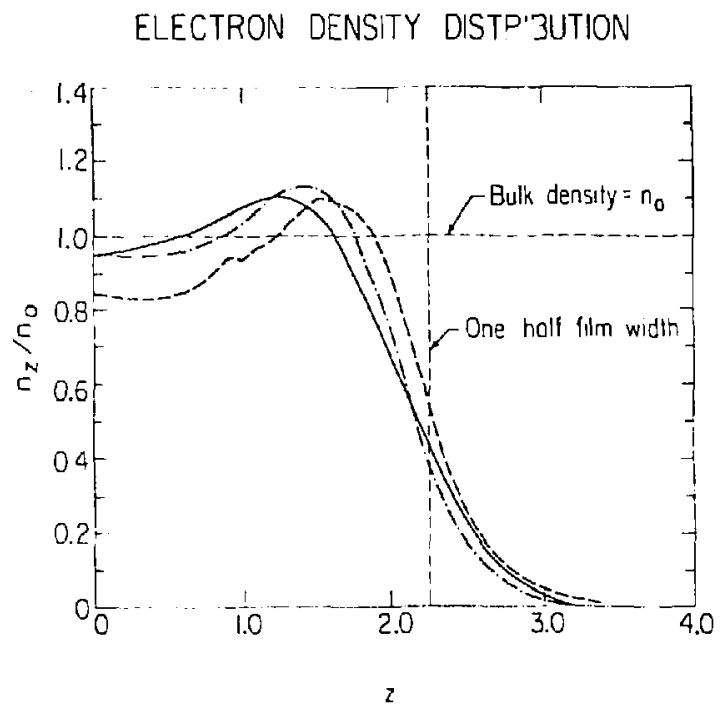

Figure 2. The electron density periendicular to a metal surface computed with variational Monte Carlo (-.-), Diffusion Monte Carlo (-.-), and density functional theory (-). Distance is in units of wigner radii.

\section{Many Body Hydrogen}

Considering the prevalence of hydrogen in the universe, and its importance in fusion research, ab initio calculation of its equation of state has advanced surprisingly little since the pioneering study of Wigner and Huntington (14), particularly in the molecular phase. Among the reasons for this lack of progress are that the hydrogen molecule distorts considerably as pressure is applied and that the zero point motion of the nuclei is much larger than in any other molecule. In fact, the molecules are almost freely rotating at low pressures and thus conventional solid state approaches run into grave difficulties.

The phase diagram of hydrogen has considerable structure, even at zero temperature. Hydrogen can be viewed as simply a two component system of electrons and protons, and as such, 
each component can undergo separate and/or correlated order-disorder transitions. At low pressures both are bound in pairs (molecules), both are however free to rotate about the center of mass of the molecule. At higher pressures ( 1 mbar) it is believed that the protons become aligned in an $\alpha-N_{2}$ lattice. At higher pressure the electrons become delocalized (molecular metal phase). At a higher pressure the protons change from a diatomic lattice to a monatomic lattice to lower their kinetic energ:" This transition probably happens at a higher pressure than the metal transition. At very high pressures $\left(1.5 \times 10^{14}\right.$ bar $)$ the protons will melt to form a two component Fermi liquid. It has been recently suggested (14) that at low pressures this melting of the proton lattice can also occur.

Hyorogen is an interesting system to study with Monte Carlo since truly ab initio calculations can be compared directly with experiment (at least in the nonrelativistic limit). Also since hydrogen has only bonding or conduction band electrons, it is the simplest and easiest case. With Monte Carlo there is no need to treat the protons differently from the electrons, they simply diffuse 1836 times slower. This does pose a difficulty since very long relaxation times can occur in a simulation. It is quite important to get an accurate trial function for the protons and to carefully prepare the initial distribution to avoid such convergence problems. This is not difficult in the moratomic solid since the protons are bound almost harmonical' to the crystal sites. However in the molecular phase, ere is much more rotational and $v i$ !rret.ional freedom and a mber of different lattice types are possible.

The Monte Carlo results are very encouraging. Figure 3 compares the Monte Carlo energies with the experimental equation of state, which is accurately known only up to 500 kbars. The trial functions used are of the Slater-Jastrow form with Gaussian orbitals for both the electrons and protons, in the molecular phase. The statistical error of systems of 108 atoms is 0.001 Rydberg/atom or $158^{\circ} \mathrm{K} / a$ tom. Monte Carlo with this trial function will be useful only at pressures such that .001 Ryd is a significant energy. This works out to be $P>10$ kbar. In the BCC solid phase the simulations agree with the best band structure calculations for a static lattice (16). The inonatomic-diatomic transition occurs at approximately 5 mbars. The proposed low density two component fermi liquid is not energetically favored confirming earlier conventional calculations with it pseudopotential (17). These results are as yet preliminary, principally because of the large amount of machine time needed for each calculation. The future will hopefully bring much better algorithms, trial functions and computers. 


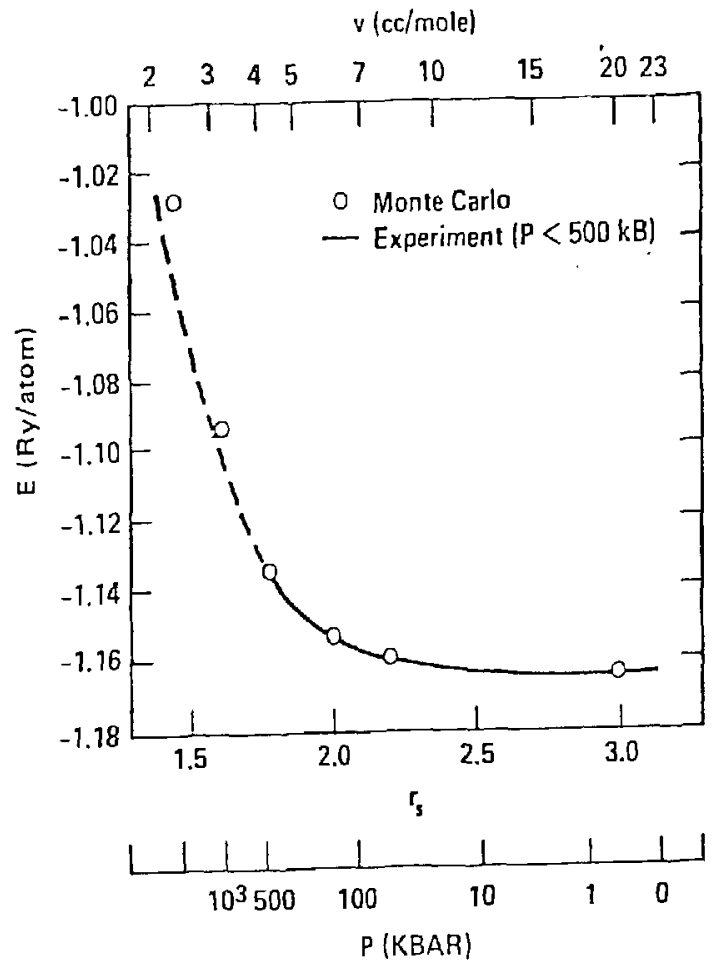

Figure 3. Ground state energy of molecular hydrogen by diffusion Monte Carlo (circles) and experiment (solid curve with dashed curve extropolation).

This work was performed under the auspices of the U. 5 . Department of Energy by the Lawrence Livermore National Laboratory under contract No. W-7405-ENG-48.

\section{REFERENCES}

1. Metropolis, N., Rosenbluth, A. H., Rosenbluth, M. N., Teller, A. M., and Teller, E.: 1953, J. Chem. Phys. 21: pp. 1087.

2. Lowther, R. E., and Coldwell, R. L.: 1980, Phys. Rev. A 22 , pp. 14 . .

3. McMillan, W. L.: 1965, Phys. Rev. A 138, pp. 442. 
4. Levesque, „.., Khiet, Tu., Schiff, D., and Verlet, L.: Orsay Report 1965 (unpublished).

5. Ceperley, D., Chester, G. V., and Kalos, M. H.: 1977, Phys. Rev. B 16, pp. 3081.

6. Ceperley, D.: 1978, Phys. Rev. B 18, pp. 3176.

7. Feynolds, P. J., Ceperley, D., Alder, B. J., and Lester, W. A.: 1982, J. Chem. Phys. 77, pp. 5593.

8. Ceperley, D., and Alder, 8. J.: 1980, Phys. Rev. Letts. 45, pp. 566.

9. Ceperley, D.: 1981, in "Recent Progress in Many-Body Theories" ed. J. G. Zabolitzky et al. (Springer-Verlag), pp. 262.

10. Ceperley, D., and Kalos,M. H.: 1979, in "Monte Carlo Methods in Statistical Physics" ed. K. Binder (Springerverlag), pp. 145.

11. Kalos, M. H., Levesque, D., and Verlet, L.: 1974, Phys. Rev. A 9, pp. 2178.

12. Ceperley, D.: 1983, J. Comp. Phys., in press.

13. Johnson, L. V.: 1982, Ph.o. Thesis, University of California, Berkeley, California, unpubiished.

14. Wigner, E., and Huntington, H. B.: 1935, '. Chrem. Phys. 3, pp.764.

15. Brouman, E. G., Kagar, Yu, and Kholas, A. K.: 1972, Sov. Phys. JETO 34, pp. 1300.

16. Ceperley, D., and Alder, 8. J.: 1981, Physica 1088, pp. 875.

17. Mon, K. K., Chester, G. V., Ashcroft; N. W.: 1980, Phys. Rev. B 2l, pp. 2641.

MSC I.MISR

This document uas prepared ab an accuunt of work spunsured by an agenc! of the I nited Statu cionernment. Vither the I nited Siates Goversment nor the

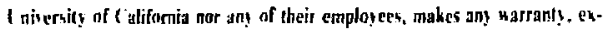
press or implied, ar aswmes any lepal liability or responsibility for the arcurars, completenews, or usefulness of any information, apparalus, product, or procms disclosed. or represents that its use would not infringe privately ouned right, Referentre therein to any specific commercial produrts. pracess, or sprs ice hy trade nanu, trademark, minulacturer, or othorwise, daes nat necresaril! constilute or insply il, condarstment, recommendatints, or fasoring by the I nited

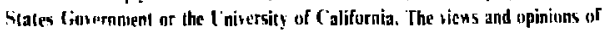
authors expressed hercin do not necessarily state or reflect thoue of the I nited states Ciosernment thereof, and shall neit be used for adrerlising or prnduct endortement purpises. 